\title{
DINÁMICA NO-LINEAL Y ECONOMÍA AUSTRÍACA
}

\author{
DULCE SAURA BACAICOA* \\ ÁNGEL RODRÍGUEZ GARCÍA-BRAZALES**
}

Resumen. En este trabajo se analizan las similitudes que existen entre ciertos rasgos del pensamiento económico de la Escuela Austriaca y algunas propiedades de la dinámica no lineal, también llamada de la complejidad. En particular, se comparan las propiedades de la teoría de la acción humana de Mises y la del orden espontáneo de Hayek, con la aparición de propiedades emergentes, autoorganización y dependencia de la trayectoria de la dinámica no lineal.

Abstract. This paper examines the similarities which exist between certain characteristics of Austrian economic thought and certain properties of nonlinear dynamics, also known as the dynamics of complexity. Specifically, the features of Mises's theory of human action and of Hayek's theory of spontaneous order are compared with the emerging properties, self-organization, and dependence which appear in the trend of nonlinear dynamics.

Palabras clave. Escuela austriaca, dinámica no-lineal, orden espontáneo, autoorganización, dependencia de la trayectoria.

Códigos JEL. B4, B5.

* Departamento de Análisis Económico, Facultad de Ciencias Económicas y Empresariales, Zaragoza

** Departamento de Análisis Económico: Teoría e Historia Económica, Facultad de Ciencias Económicas y Empresariales, Ciudad Universitaria de Cantoblanco, Madrid. Una versión más amplia de este trabajo ha sido publicada en la Revista de Economía del Rosario. 


\section{Introducción}

Este trabajo tiene por objeto comparar las notables similitudes que existen entre ciertos elementos teóricos de la Escuela Austriaca de Economía con las propiedades lógico-formales de la que vamos a denominar dinámica no-lineal. Este análisis presenta elementos de cierto interés para diversos campos del pensamiento económico. ${ }^{1}$ En primer lugar, para la historia del pensamiento económico: se demostrará que conceptos ya elaborados por Carl Menger (y anteriormente por Adam Smith) presentan rasgos de extraordinaria similitud con algunas de las propiedades de los sistemas dinámicos no-lineales. En segundo lugar, para la metodología de la ciencia en general: no es habitual que propiedades deducidas para órdenes sociales a partir de unos presupuestos metodológicos que excluyen la formalización matemática presenten rasgos teóricos similares a las deducidas, bastantes años después, por una rama de las matemáticas en estado de desarrollo incipiente. Y en tercer lugar, para la propia teoría económica: ¿es posible que la no-linealidad represente una nueva fuente de producción de teorías sin necesidad de romper con la tradición anterior? Desde luego, ciertos desarrollos de la economía evolutiva parecen apuntar en esta dirección, aunque todavía parece prematuro proponer siquiera una respuesta tentativa a esta pregunta.

En cualquier caso, y por las razones mencionadas, creemos que se trata de un análisis que reviste interés no sólo por la extraordinaria diversidad de las aproximaciones en comparación (que ya sólo esto justificaría un trabajo de esta índole), sino por la complejidad de las preguntas que suscita. Es obvio que sólo para poder llegar a un planteamiento riguroso de las mismas se precisaría un análisis detallado del alcance, implicaciones y limitaciones de las analogías científicas en general, ${ }^{2}$ trabajo que

\footnotetext{
${ }^{1}$ Se pueden encontrar algunas notas sobre este asunto en Nieto de Alba (1988).

${ }^{2}$ La literatura sobre este asunto ha aumentando notablemente en los últimos años a raíz de la publicación del trabajo de Phillip Mirowski More Heat than Light:
} 
no se va a realizar aquí. Más bien, de lo que vamos a tratar aquí es de un caso especial y peculiar: una situación en la cual dos teorías diferentes, sin buscar deliberadamente la analogía, deducen por separado una serie de propiedades que, desde un punto de vista estrictamente analítico, son análogas. Se trata, si se nos permite abusar del lenguaje, de un trabajo «empírico» dentro del planteamiento general del problema que, esperemos, pueda servir de ayuda para tratamientos más sistemáticos.

El análisis se desarrollará en dos fases: en primer lugar, se presentarán las características generales y algunas de las implicaciones formales y teóricas más importantes de la dinámica nolineal tanto para la ciencia en general como para la economía en particular. Hemos de tener presente que los conceptos de autoorganización, propiedades emergentes, dependencia de la trayectoria, heterogeneidad y no equilibrio, se han ido formulando no sólo como propiedades formales de los sistemas dinámicos no lineales, sino también como propiedades de fenómenos observados en el mundo físico. El descubrimiento de estas nuevas propiedades del mundo físico han tenido su aplicación al estudio de los fenómenos humanos y, en particular, los económicos, proceso éste que ha tenido lugar principalmente en escuelas de pensamiento donde la formalización es considerada como algo propio de la teoría económica.

En segundo lugar, se procederá al análisis objeto de este artículo, esto es, comprobar si ciertas propiedades de los sistemas dinámicos no lineales aparecen ya enunciadas en los textos clásicos de la doctrina austriaca. Para ello, hemos decidido centrar la búsqueda alrededor de tres campos: la irreversibilidad de los

Economics as Social Physics, Physics as Nature's Economics, Cambridge University Press, Cambridge y Nueva York, 1989. Sin embargo, no se debe olvidar que durante la segunda mitad del siglo XIX, tanto destacados teóricos de la economía como Carl Menger, Vilfredo Pareto, León Walras, Alfred Marshall o Gustav Schmoller, como otros muchos teóricos sociales, Paul von Lilienfeld, Albert Schäffle o Augusto Comte ya realizaron profundas reflexiones sobre este asunto. 
procesos sociales y económicos, las propiedades de los órdenes espontáneos (propiedades emergentes y no-equilibrio) y la $d e-$ pendencia de la trayectoria.

\section{La dinámica no-lineal}

En los últimos 40 años se ha producido, en diversos campos científicos, una importante evolución conceptual y metodológica ligada al análisis de los fenómenos dinámicos desde un enfoque no lineal; este análisis se engloba, al menos en parte, en la denominada ciencia de la complejidad o teoría dinámica de los sistemas complejos. Este enfoque ha puesto de manifiesto distintas propiedades comunes a fenómenos físicos, químicos o biológicos y abre nuevas posibilidades en el camino de reducir las barreras interdisciplinares y avanzar en la solución de problemas existentes hace tiempo en diversas disciplinas. Esta nueva concepción, según el premio Nobel de química Ilya Prigogine, «es una reconceptualización de la ciencia, tanto en el campo de las ciencias duras como en el de las ciencias sociales. Por todas partes la visión de la naturaleza se dirige hacia lo múltiple, lo temporal y lo complejo» (Prigogine 1993: 3).

Estudios asociados a disciplinas como la física de la atmósfera, termodinámica de los procesos irreversibles, matemática de los sistemas no lineales, electromagnetismo, ciencia de los materiales, etc., han desafiado los límites establecidos tradicionalmente entre orden y desorden, determinismo y aleatoriedad, inestabilidad y comportamiento explosivo. La dinámica compleja ha puesto de manifiesto que propiedades como la irreversibilidad, la inestabilidad o la dependencia de la trayectoria - path dependency - son comunes al comportamiento de fenómenos dinámicos en campos científicos muy diversos.

Una primera aproximación al estudio de los sistemas complejos nos presenta un conjunto de características y conceptos, 
no todos claramente definidos, que van conformando un bloque que algunos denominan ciencia de la no linealidad, y otros, ciencia de la complejidad o teoría de los sistemas complejos. La consistencia de este bloque no está exenta de ciertas ambigüedades, comprensibles, por otra parte, en un proceso científico aún en desarrollo. Sin embargo, y pese a esta falta de definición que presenta en ocasiones, no podemos ignorar que sus principios teóricos y sus aplicaciones ocupan un lugar cada día más importante en el desarrollo de diversas disciplinas científicas.

Una de las consecuencias más importantes de este nuevo enfoque dinámico es que exige contemplar los fenómenos en una doble perspectiva: por una parte, no es posible comprender el resultado de un proceso sin analizar sus componentes y la forma en que éstos se relacionan; por otro lado, tampoco es posible deducir, del análisis individualizado de estos componentes, las propiedades del conjunto del sistema. Por tanto, si admitimos que los sistemas económicos son sistemas complejos y la dinámica compleja puede permitirnos avanzar hacia su comprensión, deberemos cuestionar determinados conceptos y aproximaciones teóricas así como profundizar en aquellas que han sido capaces de, al menos, considerar algunas de estas propiedades.

Como punto de partida, consideremos la noción de complejidad propuesta por S. Auyang: «Los sistemas complejos son sistemas capaces de autoorganizarse; están constituidos por muchos componentes que interaccionan entre sí y presentan muchas estructuras a diferentes escalas; subyacen muchos procesos dinámicos a diferentes velocidades y tienen la capacidad de cambiar bruscamente y adaptarse a su entorno» (Auyang 1988: 13).

Frente a esta concepción de la complejidad como producto, entre otros factores, de la presencia de múltiples componentes que interactúan entre sí, autores como I. Prigogine afirman que las características del comportamiento de estos sistemas se derivan de que son sistemas abiertos que presentan inestabilidades y comportamientos alejados del equilibrio, donde se generan 
propiedades imposibles de explicar a partir de sus componentes considerados individualmente. Esto es, son capaces de adaptarse y generar nuevas estructuras en situaciones de desequilibrio, es decir, generan propiedades emergentes.

Así que, mientras todos los autores consultados están de acuerdo en que la propiedad de autoorganización es una característica esencial de estos sistemas, unos y otros centran su interés en otros rasgos (interacciones locales, irreversibilidad, etc.), que, a su juicio, permiten avanzar en la explicación de su comportamiento.

Vamos a considerar ahora algunos conceptos clave de la dinámica no-lineal, con el fin de analizar si éstos han sido o no desarrollados por las diversas escuelas de pensamiento económico. En particular, consideraremos los conceptos de autoorganización, propiedades emergentes, dependencia de la trayectoria, heterogeneidad y no equilibrio.

Comenzaremos por el primer concepto, esto es, por la idea de autoorganización. Este concepto se utiliza normalmente para designar la capacidad de un sistema para generar a partir de sus componentes en interacción, características que no pueden encontrarse en esos componentes considerados individualmente. Es decir, aparecen las denominadas propiedades emergentes; no todas las propiedades de un sistema son propiedades emergentes. Algunos autores distinguen entre propiedades resultantes, que son esencialmente similares a las propiedades de los constituyentes y pueden obtenerse por agregación o generalización a partir del comportamiento de los componentes, y propiedades emergentes, que son específicas del sistema considerado como un todo, y que son el resultado de procesos dinámicos de interacción entre las partes y de adaptación a las perturbaciones del entorno. La autoorganización puede desarrollarse espacial o temporalmente, mantener o no propiedades estables y no debe, en todos los casos, entenderse como sinónimo de mayor orden. 
En segundo lugar, se señala que estos procesos están fuertemente condicionados por su historia, esto es, que son dependientes de la trayectoria. Cualquier factor que discrimine a favor de un cierto comportamiento en un momento determinado puede seleccionar comportamientos futuros, aunque estos pudieran ser considerados, a priori, menos eficientes.

En las ciencias sociales, señala Rizello, «este concepto implica que cualquier acto sucesivo en el desarrollo de un individuo, una organización o una institución está fuertemente influenciado y depende de la trayectoria (experiencia y evolución) previa» (Rizello 1997: 100).

La idea subyacente es que la estructura de las organizaciones se deriva de un proceso condicionado por su propia historia. Los procesos dinámicos con esta característica se dice que presentan dependencia de la trayectoria. Esta propiedad es especialmente interesante en el caso de que, por determinadas restricciones debidas bien a factores externos o a deficiencias iniciales (por ejemplo, carencias de información), el sistema selecciona estados considerados inferiores a otros; a partir de una determinada situación inicial, se pueden poner en marcha mecanismos de reforzamiento y procesos dinámicos irreversibles que imposibiliten de forma definitiva el acceso del sistema a estados más eficientes. Como señalan J, Groenewegen y J. Vromen,

«dependencia de la trayectoria es una idea opuesta a la idea tradicional de que fuerzas o mecanismos sistemáticos dirigen el desarrollo económico hacia una única posición de equilibrio eficiente» (Groenewegen y Vromen 1997: 49).

Finalmente, la heterogeneidad y la diversidad entre los elementos constituyentes del sistema es propiedad destacada por muchos investigadores en este campo; algunos lo señalan, incluso, como una condición necesaria para la aparición de propiedades emergentes (Auyang 1998). En relación con el análisis económico Arrow escribe: «El supuesto de homogeneidad me parece especialmente peligroso. Niega el supuesto fundamental 
de la economía, que es que está construida sobre las ganancias del comercio que surgen de las diferencias individuales. Además, desvía la atención de un aspecto muy importante, a saber, los efectos de la distribución de la renta y de otras características individuales y del funcionamiento de la economía» (Arrow 1987: 71).

La consideración conjunta de todos estos conceptos nos sitúa claramente en el marco del análisis no-lineal. Aquí, el conjunto no es la suma de sus partes ni sus propiedades se pueden obtener agregando las características de sus componentes. Una pequeña perturbación puede desencadenar efectos de gran envergadura, mientras que un sistema sometido a grandes perturbaciones puede mostrar estructuras más ordenadas, contrariamente a lo que podría suponerse. Estas consideraciones conducen a algunos autores a afirmar que la no-linealidad es el elemento esencial de un nuevo enfoque dinámico, tanto en las ciencias naturales en general, como en la economía en particular.

De lo expresado hasta ahora parece deducirse que, con formalización matemática o sin ella, los procesos dinámicos que corresponden al dominio de la Economía tienen características que les hacen adecuados, en principio, para ser explicados en el marco de la dinámica de los sistemas complejos: muchos componentes en interacción que se comunican, que imitan y aprenden, que son capaces de corregir sus comportamientos, adaptarse a su entorno y modificarlo y generar a través del tiempo nuevas estructuras y restricciones. Sin embargo, deberíamos ser prudentes y abrir una profunda reflexión acerca del alcance y utilidad de la consideración de conceptos y técnicas propios de las ciencias naturales en Economía. Hay autores que se muestran especialmente preocupados por la dificultad que representa el hecho de que la economía trata de problemas y decisiones relativas al ser humano: «Lo fundamental es la diferencia que surge del hecho de que está implicada la inteligencia humana: las unidades microscópicas de los sistemas físico-químicos no modifican 
su comportamiento como consecuencia de un aprendizaje sistemático ni de pensamientos y razonamientos estratégicos, no innovan, es decir no crean conscientemente nuevas formas de comportamiento» (Witt 1985: 572).

Sin embargo, aun conscientes de estas limitaciones, hay autores que consideran que es más importante el avance que representa la dinámica no-lineal porque permite superar las limitaciones que supone el esquema neoclásico. Esto nos permitiría abrir caminos a la incorporación de conceptos que supongan una ruptura con conceptos como el equilibrio, la optimización y la estabilidad. $\mathrm{Si}$, además, se propone una modelización formal que aún pueda permitirnos avanzar en la comprensión de los fenómenos económicos, y generar resultados más acordes con el mundo real, parece claro que debemos intentar superar la dinámica lineal. Sin embargo, comprobaremos que no es suficiente un planteamiento no-lineal para que la modelización planteada pueda recoger las características más importantes de la dinámica de los procesos económicos si éstos son planteados como sistemas complejos.

Es evidente que la dinámica no-lineal plantea conceptos y resultados interesantes que abren nuevas posibilidades para formalizar fenómenos que quedaban hasta hace poco en el campo de lo aleatorio; la matemática del caos nos permite obtener dinámicas que presentan cambios esenciales ante pequeñas perturbaciones (bifurcaciones) y considerar cómo sistemas muy simples y deterministas pueden presentar un comportamiento aparentemente aleatorio y ser impredecibles a medio y largo plazo debido a la propiedad denominada «sensibilidad a las condiciones iniciales». La matemática del caos nos enseña también que un sistema caótico no es sinónimo de un sistema «desordenado», sino que existen estructuras y pautas ordenadas subyacentes al caos.

En economía, el planteamiento no lineal y la aplicación de las matemáticas del caos han enriquecido notablemente algunos 
campos, y eso a pesar de la resistencia que la ciencia económica, al contrario que otras disciplinas, está presentando a la consideración de los nuevos conceptos. La aplicación de la modelización no-lineal en economía no es nueva ni tampoco escasa. Ya hace más de 50 años algunos autores propusieron que debíamos aproximarnos al fenómeno del ciclo económico con un planteamiento no lineal (Kalecki, 1935; Goodwin, 1947; Kaldor, 1956). Sin embargo, como señala M.W. Lorenz, «parece como si los fenómenos no lineales se hayan incorporado en otras disciplinas con más aceptación que en economía» (Lorenz 1993: 21). Aunque puede argumentarse que siempre las nuevas técnicas y conceptos necesitan un periodo de tiempo más o menos amplio para ser considerados en otras disciplinas, resulta difícil de aceptar que una ciencia que alcanza su mayor desarrollo importando conceptos de la Física y generalizando el uso de las técnicas analíticas, se resista, como mínimo, a la consideración de la renovación conceptual de las disciplinas que le sirvieron de referencia en sus intentos de constituirse como disciplina científica. Debemos analizar si hay otras razones que nos ayuden a entender la concentración sobre los sistemas dinámicos lineales en economía.

Es evidente que hay una razón importante, y es la simplicidad; si las propiedades del fenómeno que consideramos no se ven alteradas esencialmente si los tratamos como lineales, y supuesto que todos nuestros modelos son siempre aproximaciones y simplificaciones, está justificado un tratamiento lineal; la cuestión importante es que, en un primer análisis, sabemos que hay grandes diferencias en los resultados que ofrecen las dos formulaciones y que en la realidad parece más general la presencia de fenómenos complejos.

Hay otra razón quizás más importante, señalada por autores como Lorenz y Mirowski: el mantener nuestro ideal de ciencia

3 R. Goodwin trabajó a lo largo de su vida en numerosas aportaciones utilizando la aproximación no lineal. Es de destacar su aportación a la explicación del ciclo económico. 
en el paradigma de la mecánica clásica, e intentar representar sistemas con comportamientos predecibles y regulares con la idea básica de un equilibrio dinámico estable.

«De acuerdo con la analogía con la Mecánica clásica, la mecánica de la utilidad y el interés propio restablece el equilibrio anulando el potencial libre en los mercados (o sea, los deseos de revisar los planes individuales no satisfechos); cuando esto ocurre se alcanza el estado de equilibrio y fue este estado a cuyo estudio se dedicaron la mayoría de los esfuerzos teóricos. En vez de analizar cómo se elimina ese potencial (o sea investigar la complicada dinámica de las interacciones que son la causa de la coordinación), sólo se consideró el estado final»

(Witt 1985: 573).

Esta analogía con la mecánica, según Witt, «sesga profundamente las preconcepciones del investigador respecto de la dinámica» (Witt 1985: 574). A partir de esto, podemos comprender que una modelización que demuestra la imposibilidad de predicción y que cuestiona los conceptos mismos de equilibrio y estabilidad, sea considerada con mucho recelo entre los economistas. Un ejemplo significativo de este hecho es la explicación del ciclo económico de la denominada teoría de los ciclos reales. Esta teoría se enfrenta con un problema habitual dentro del análisis económico: los datos no presentan la clase de regularidad que cabría esperar de sistemas deterministas lineales. La divergencia se resuelve superponiendo, a una estructura básicamente lineal, perturbaciones estocásticas exógenas. De esta forma, su investigación tiene por objeto encontrar un conjunto de valores para los parámetros y para determinadas características de las perturbaciones que permitan replicar las series empíricas. $Y$, sin embargo, una modelización no-lineal determinista que genere resultados similares y que contenga un mayor poder explicativo es rechazada, entre otras razones, porque presenta el problema de la imposibilidad de predicción a medio y largo plazo. Así podemos entender por qué la aproximación no lineal en la dinámica económica se ha considerado, principalmente, por econo- 
mistas que de una forma u otra no participan de la idea del paradigma clásico del equilibrio económico.

Para terminar esta breve presentación, deberíamos preguntarnos si la mera consideración de relaciones matemáticas no lineales es en sí un avance hacia la consideración de los fenómenos económicos como fenómenos complejos. ¿Es la no-linealidad la característica fundamental de lo que hemos denominado sistemas complejos?

Para reflexionar sobre esto, pongamos un ejemplo: supongamos un modelo de crecimiento donde, bajo la consideración de la familia representativa, respetando las hipótesis habituales sobre expectativas (expectativas racionales o previsión perfecta) y la homogeneidad de los agentes, consideramos formas funcionales no lineales ni linealizables; sabemos que es posible que obtengamos dinámicas más complicadas y resultados de interés sobre la posible existencia de ciclos o, incluso, dinámicas caóticas. Pero, a partir del planteamiento inicial, y con independencia de los resultados que obtengamos, podemos plantearnos algunas cuestiones: ¿Es compatible la constancia en el tiempo de características estructurales como tecnología y/o preferencias, la consideración de homogeneidad de los agentes, la no interacción entre ellos, etc., con la consideración del sistema económico como un sistema con capacidad de autoorganización y con propiedades emergentes capaces de modificar su estructura a lo largo del tiempo? ¿Y qué decir sobre cómo planteamos la visión dinámica que tienen los agentes de las posibles consecuencias de sus acciones? Es decir, ¿podemos aceptar la compatibilidad con la hipótesis de expectativas racionales? Por tanto, nos tenemos que preguntar si, con independencia del tipo de formalización matemática escogida, cualquier tratamiento del problema es coherente con los principios de la dinámica compleja.

Cuando más se profundiza en la teoría dinámica de los sistemas complejos y observamos la estructura social y los fenómenos económicos desde ésa óptica, comprendemos qué lejos se 
encuentra la formalización teórica de los modelos económicos de un tratamiento compatible con esa visión. Y no se trata de que el tratamiento sea incompleto, ni que la necesidad de la sencillez para explicar induzca una aproximación limitada a una dinámica compleja; por el contrario, se considera que la linealidad, la homogeneidad de los agentes, la constancia de preferencias y del marco social, el cuasi-perfecto conocimiento de la información sobre el futuro que los agentes necesitan para decidir, etc., son conceptos e hipótesis adecuados para explicar la realidad económica. $Y$, lo que es peor, no parece ser una etapa provisional donde, aceptando las limitaciones, se percibe la necesidad de avanzar hacia otra conceptualización nueva; por el contrario, se percibe en las líneas principales de desarrollo de la economía dinámica una aceptación cuasi-general de esos principios y una postura defensiva frente a nuevos planteamientos.

Hay algunas excepciones a esa aparente aceptación generalizada de la dinámica neoclásica; por ejemplo, la denominada Economía Evolutiva, que parte de un cambio de ciencia de referencia de la mecánica clásica hacia la biología y constituye un intento por estudiar los procesos dinámicos (difusión de innovaciones, crecimiento económico y dinámica industrial entre otros) se propone ser una alternativa a la economía neoclásica y a su enfoque dinámico. No es objeto este trabajo una revisión amplia de las aportaciones de esta corriente teórica, pero, con el objeto de señalar algunos avances respecto a otros planteamientos, se pueden destacar determinados rasgos: la heterogeneidad de los agentes y de las organizaciones como elemento clave que permite la evolución y la selección y la consideración de la incertidumbre como un componente inevitable del proceso dinámico. ${ }^{4}$ A pesar de constituir un conjunto heterogéneo, los

\footnotetext{
${ }^{4}$ Estos son sólo algunos de los rasgos fundamentales. Junto a esto se debe destacar el principio de racionalidad limitada, que justifica los comportamientos rutinizados y la posibilidad de imitación, aprendizaje e innovación así como la formulación de procesos dinámicos dependientes de la trayectoria.
} 
autores que forman la economía evolutiva consideran, de forma general, como sus predecesores a Schumpeter y Marshall, el institucionalismo y a la Escuela Austriaca; y dentro de ésta última, destaca sobre el resto de autores la aportación de Hayek. Aunque algunos de estos autores ${ }^{5}$ señalan ciertos problemas asociados al principio del individualismo metodológico de la Escuela Austriaca, todos admiten que la visión dinámica de esta escuela teórica es un punto de referencia básico para sus desarrollos.

No vamos a servirnos, sin embargo, de este nexo de unión para tratar las similitudes entre determinadas propiedades de la dinámica no-lineal y la Escuela Austriaca. Entre otras muchas razones, porque, como ya hemos señalado anteriormente, la dinámica compleja tiene unas características que son previas a la elección del tipo de planteamiento formal (o el rechazo del mismo) que elijamos para nuestra explicación. Nuestro objetivo es revisar la aportación de aquellos autores de la Escuela Austriaca que plantean una visión dinámica más próxima a la dinámica compleja y seleccionar qué contenidos pueden señalarse que permitan contestar a la cuestión de si nos encontramos en el marco de una teoría dinámica de los sistemas complejos.

\section{Economía Austriaca y propiedades de la dinámica no lineal}

Como ya avanzábamos en la introducción, las similitudes, alusiones y referencias no tanto a la no-linealidad cuanto a los fenómenos asociados a ésta, aparecen con relativa frecuencia en la literatura asociada genéricamente con la Economía Austriaca. El fenómeno resulta más notable, si cabe, por cuanto se trata de una escuela de pensamiento económico que, como es bien sabido, renunció desde sus comienzos a la formalización matemática

\footnotetext{
5 Entre otros, G. Hodgson en Economía y Evolución (1995)
} 
como método de expresión de sus leyes y modelos económicos. Sin embargo, y como se demostrará en este apartado, no cabe entender estas similitudes y alusiones sólo como meras «coincidencias». Resulta que ciertos fenómenos asociados a la no-linealidad constituyen un elemento nuclear en la estructura teórica desarrollada por la Escuela Austriaca.

Sin embargo, antes de referirnos de forma explícita a los fenómenos en cuestión y al papel que juegan en el pensamiento austriaco, hemos de precisar ciertos aspectos metodológicos - a efectos de esta investigación- de tal forma que no quede lugar a dudas de la fiabilidad de las fuentes consultadas. En primer lugar, hay que señalar que mientras la investigación de ciertos fenómenos, como el surgimiento de órdenes espontáneos, constituyen en sí mismos una de las señas de identidad de esta escuela de pensamiento, otros, como la irreversibilidad y la flecha del tiempo, apenas si aparecen enunciados y, en el caso de estarlo, se trata de frases aisladas intercaladas en los más diversos contextos. En segundo lugar, hemos de precisar que todos estos conceptos se desarrollaron sin referencias ni citas explícitas a lo que hoy en día entendemos por no-linealidad. No quiere esto decir que, al menos en el caso de Hayek, se puedan aportar citas y testimonios que apunten a que este autor tenía cierto conocimiento de los desarrollos contemporáneos de la teoría de sistemas, por ejemplo. ${ }^{6}$ Pero, al menos a lo que alcanza nuestro conocimiento, no existe evidencia de que esto sea así. En tercer lugar, hay que señalar que lo que nos proponemos aquí no es aportar evidencia documental de si se puede relacionar o no a la Escuela Austriaca con lo que de un modo general se denomina nolinealidad. Todo lo contrario; lo que pretendemos demostrar es que del propio pensamiento austriaco se desprende directamen-

${ }^{6}$ Sólo por poner un ejemplo, en el prefacio de The Sensory Order, podemos leer: «[Agradezco] a mis amigos Karl R. Popper y L. von Bertalanffy y al Profesor J.C. Eccles por haber leído y comentado las anteriores redacciones de este libro» (Hayek 1952: viii-ix). 
te una forma de teorizar acerca de los fenómenos sociales y económicos que guarda evidentes analogías con conceptos desarrollados por la no-linealidad.

La razón de esta afinidad tan insospechada reside, entre otras muchas razones, en el papel crucial que juega un determinado concepto de la acción humana en la teoría económica de la Escuela Austriaca y, en particular, en una propiedad de las consecuencias generadas por la acción humana: la irreversibilidad de sus resultados.

Comenzaremos por una breve exposición del concepto de la acción humana en Ludwig von Mises, ya que es aquí donde se pone de manifiesto, no sólo el carácter irreversible de la mayoría de las acciones humanas, sino también el surgimiento de órdenes espontáneos en el seno de las sociedades así como el carácter evolutivo de éstos.

\subsection{La acción humana como proceso irreversible}

Ludwig von Mises y Friedrich A. Hayek son los teóricos de obligada referencia para entender el núcleo doctrinal de la Escuela Austriaca. Ambos sentaron las bases de la doctrina moderna en sus más diversas extensiones y fueron los maestros directos de la primera generación de teóricos neoaustriacos de origen norteamericano - entre ellos, Israel Kirzner y el recientemente fallecido Murray N. Rothbard-. Pero aunque todos ellos han realizado importantes contribuciones, la caracterización más sistemática del agente económico de la Escuela Austriaca la encontramos en La Acción Humana de Ludwig von Mises (Mises 1995). Aquí se nos presenta un agente económico bastante más complejo que el llamado homo oeconomicus neoclásico. Para empezar, se localiza el comportamiento económico de la persona como parte de una acción humana que, entre otras múltiples propiedades, va a tener una que nos interesa especialmente: la de ser irreversible. 
Por acción humana entiende Mises todo proceder consciente y deliberado. Este proceder aspira a sustituir un estado menos satisfactorio por otro mejor. Pues bien, este proceder se puede esquematizar en términos de la obtención de un fin o fines —estado de la realidad que se quiere alcanzar- recurriendo al uso de unos medios que, por definición, van a ser escasos. ${ }^{7}$ Podemos avanzar ya, a partir de este esquema de medios-fines, que el comportamiento económico no es sino un aspecto más de la acción humana, ${ }^{8}$ y que las propiedades de la acción humana se «transmiten», por decirlo de alguna forma, al comportamiento económico. En particular, y como tendremos oportunidad de ver a lo largo de este apartado, también se «transmite» la irreversibilidad.

Una vez que los agentes han formulado sus esquemas de medios-fines, formulan planes de acción mediante los cuales pretenden llevar a cabo la modificación de la realidad buscada. Estos planes de acción, con entera independencia de que puedan ser llevados a cabo o no, se formulan de acuerdo con dos principios:

a) el principio de causalidad: la búsqueda de una relación causal, de carácter recurrente, que opera en la parte del mundo sobre la que vamos a ejercer nuestra acción;

b) el principio teleológico: el hombre actúa en función de los fines que pretende alcanzar, de las metas que se impone.

Pues bien, precisamente de estos dos principios se desprende la propiedad de irreversibilidad de la acción humana, en la medida en que su operación en la formulación de los planes impli-

${ }^{7}$ Los requisitos previos de constitución de toda acción humana son, a juicio de Mises, tres: a) Representación de un estado de la realidad: cambiar un estado de la realidad a través de la acción implica una representación previa de un estado más satisfactorio que el vigente en ese momento; b) Debe concurrir la voluntad consciente de cambiar un estado de cosas por considerarlo insatisfactorio; y c) Debe percibirse una conducta deliberada capaz de cambiar el estado de la realidad en cuestión.

8 Y que la economía no es más que una disciplina entre otras muchas pertenecientes a una teoría general de la acción humana. 
can un «antes» y un «después». El propio Mises lo entiende así: «Anterioridad y consecuencia son conceptos esenciales al razonamiento praxeológico y lo mismo sucede con la irreversibilidad de los hechos» (Mises 1995: 120).

Este es uno de los pasajes en los que más claramente Mises habla de la irreversibilidad del paso del tiempo. Pertenece al capítulo V de La Acción Humana, en el cual se analiza, precisamente, el papel que desempeña el tiempo en las acciones humanas. Es de este capítulo del que se puede extraer una idea más precisa del carácter irreversible de la acción humana tal y como la entiende Mises, pese a que no volveremos a encontrar en el mismo una afirmación tan explícita como la anterior. Sin embargo, la mera operación de los principios de causalidad y teleológico ilustran el sentido en que Mises entendía el fenómeno de la irreversibilidad del tiempo y de la historia. Dos son, a nuestro juicio, las referencias clave: «La acción influye exclusivamente sobre el futuro; nunca sobre un presente que, con el transcurso de cada infinitesimal fracción de segundo, va inexorablemente hundiéndose en el pasado. El hombre adquiere conciencia del tiempo al proyectar la mutación de una situación actual insatisfactoria por otra más atrayente» (Mises 1995: 120). «El presente, en cuanto duración temporal, equivale a la permanencia de unas precisas circunstancias. Cada tipo de actuación supone la concurrencia de condiciones específicas, a las que hay que amoldarse para conseguir los objetivos perseguidos. El presente praxeológico, por lo tanto, varía según los diversos campos de acción; nada tiene que ver con el paso del tiempo astronómico. El presente, para la praxeología, comprende todo aquel pasado que todavía conserva actualidad, es decir idoneidad para la acción...» (Mises 1995: 121).

Aunque estos dos pasajes hagan referencia al carácter proyectivo de la acción y al concepto de tiempo praxeológico, se deja ver claramente - sobre todo en el primero- que Mises entiende el pasado de la acción como algo definitivo y no reversible, aunque 
sí modificable. Una acción orientada al futuro opera sobre una realidad que, a cada instante que transcurre, es pasado. Pasado que se aspira a modificar, pero desde el cual el agente no volverá a operar. En este sentido, el presente praxeológico, concepto mucho más amplio que el presente analógico, ha de entenderse como continuidad de la acción en el tiempo. La mutación de las circunstancias a las cuales la acción tiene que amoldarse a medida que poco a poco el presente praxeológico va transcurriendo, implica que no es posible volver a la posición previa a al inicio de la acción y rectificar juicios erróneos. El agente tendrá que actuar con las circunstancias dadas de su presente praxeológico, tanto si son las previstas como si no lo son.

Como se ve, no se puede decir que Mises tratara de forma sistemática el fenómeno de la irreversibilidad. Sin embargo, se observa claramente que daba por sentado que la acción humana y el tiempo praxeológico — que es el tiempo humano-son irreversibles y que, por tanto, actuar implica en muchas ocasiones adaptarse a cambios no previstos en la realidad relevante para nuestra acción y que, por tanto, la acción no puede retrotraer la realidad a la situación exacta en que se encontraba antes de los cambios. En este sentido, podemos decir que Mises no era un teórico de la irreversibilidad: simplemente la daba por descontada.

\subsection{El orden espontáneo}

A diferencia del análisis de la irreversibilidad de la acción humana, el análisis de los órdenes espontáneos en la obra de los economistas austriacos, y en particular en la de Friedrich A. Hayek, es mucho más inmediata. Hayek puede considerarse como el gran artífice de una teoría general del orden social cuya característica más importante es el carácter espontáneo del mismo. ${ }^{9}$

9 Hayek insiste mucho en el carácter espontáneo del orden social en su obra: «Para captar adecuadamente el íntimo contenido del orden que caracteriza a la sociedad civilizada, conviene advertir que este orden, lejos de ser fruto de designio 
Pero para entender esta espontaneidad, será necesario en primer lugar hacer algunas aclaraciones sobre lo que Hayek entiende por orden social y por evolución del mismo.

El orden social es entendido por Hayek a partir de un conjunto de normas que permiten la supervivencia de poblaciones que superan el ámbito familiar o tribal. ${ }^{10} \mathrm{Al}$ entender así el orden social, Hayek diferencia por completo dos tipos de órdenes sociales que pueden coexistir en el tiempo, aunque no de forma estable: el orden tribal, basado en comportamiento instintivo, válido para asegurar la supervivencia de pequeñas comunidades, y el orden extenso, un conjunto de normas generadas a través de un proceso de selección social — de carácter distinto al natural一, y que permite la supervivencia pacífica y cooperante de grupos humanos de gran tamaño. La superioridad del orden extenso queda puesta de manifiesto, según Hayek, por el

o intención, deriva de la incidencia de ciertos procesos de carácter espontáneo» (Hayek 1997: 189). «El mensaje fundamental que he intentado transmitir a lo largo de mi argumentación queda reflejado en mi insistencia en el carácter meramente espontáneo de las normas que facilitan la formación de estructuras que disponen de la capacidad de auto-organizarse» (Hayek 1997: 231-232). «Fueron en gran medida estos esfuerzos orientados a la comprensión de la formación de la interacción humana mediante la evolución y la espontánea estructuración de un orden lo que convirtieron estos planteamientos en el principal instrumento para tratar esos fenómenos complejos para cuya explicación no sirven las «leyes mecánicas» de causación unidireccional... (Hayek 1997: 375).

10 «Son las normas reguladoras del humano comportamiento, plasmadas por vía evolutiva (y especialmente las que hacen referencia a la propiedad plural, al recto comportamiento, al respeto de las obligaciones asumidas, al intercambio, al comercio, a la competencia, al beneficio y a la inviolabilidad de la propiedad privada), las que generan tanto la íntima estructura de ese peculiar orden como el tamaño de la población actual. Tales esquemas normativos se basan en la tradición, el aprendizaje y la imitación más que en el instinto y consisten fundamentalmente en un conjunto de prohibiciones («no se debe hacer tal cosa») en virtud de las cuales quedan especificados los dominios privados de los distintos actores. La humanidad accedió a la civilización porque fue capaz de elaborar y de transmitir -a través de los procesos de aprendizaje- esos imprescindibles esquemas normativos (inicialmente limitados al entorno tribal, pero extendido más tarde a espacios cada vez más amplios) que, por lo general, prohibían al hombre ceder a sus instintivas apetencias y cuya eficacia no dependía de la consensuada valoración de la realidad circundante» (Hayek 1997: 198-99). 
mayor tamaño del grupo humano que vive actualmente bajo ese sistema de normas, respecto del grupo que vive bajo el sistema tribal. La garantía de éxito no viene dada, sin embargo, por el éxito demográfico. Éste constituye, más bien, una consecuencia del proceso evolutivo.

¿Cuál es, entonces, el tipo de evolución de las normas? En la medida en que este proceso implica necesariamente una transmisión generacional de los hábitos adquiridos - lo que no se observa en el mundo natural- es fácil comprender que Hayek no entienda la evolución social de forma darwinista. Precisamente es en los procesos de propagación de las normas «superiores» donde se aprecia más claramente el carácter espontáneo del orden social. Las normas se transmiten a través de un proceso de selección de aquellas más eficientes para la supervivencia de la comunidad o sociedad en cuestión. Sin embargo, a partir de un determinado momento de su desarrollo - que puede ser posterior a la desaparición de la comunidad humana donde se originó la norma- la razón o el fin último para el cual aquella norma o pauta de comportamiento surgió, se olvida y simplemente se utiliza la norma en cuestión porque, sin saberlo, garantiza la pervivencia del grupo humano que la adopta. La espontaneidad radica en que el proceso de imitación o de aprendizaje de la norma no es diseñado por nadie, ni se atiene ya a la finalidad para la que fue diseñada. La inserción de esta vieja norma en un esquema «nuevo» de normas da lugar a un orden social distinto al anterior. El tránsito de un orden a otro es espontáneo en la medida en que, en primer lugar, no es previsible el resultado final y, en segundo lugar, depende de la disposición de las personas pertenecientes a esa comunidad su adopción o no. Si el proceso de adaptación y de propagación no es satisfactorio, ese grupo humano desaparece en el registro de la historia. Si el proceso es satisfactorio, el grupo pervive y, con él, la norma tradicional. Se entendería así la extraordinaria complejidad de los actuales órdenes sociales, con herencias culturales muy 
diversas, con rasgos extraordinariamente similares a los de órdenes pasados pero auto-organizando sociedades de forma radicalmente distinta. ${ }^{11}$

Esto es, a grandes rasgos, lo que entiende Hayek por un orden social espontáneo. Aunque sólo fuera por esta teoría, sería pertinente incluirla en nuestro análisis. Pero hay más. Consciente como era de las dificultades metodológicas a que este tipo de teoría daba lugar, Hayek tuvo que realizar un enorme esfuerzo para precisar exactamente qué es lo que entendía por una teoría que fuese capaz de explicar pautas de comportamiento de un sistema - surgimiento de órdenes-, pero que no fuese capaz de generar predicciones con el grado de precisión que se había alcanzado en la física. Este esfuerzo aparece reflejado en diversos lugares de su vasta producción científica. Pero donde podemos encontrar un tratamiento específicamente destinado a entender en qué consiste el estatuto metodológico y científico de este tipo de teoría es en tres trabajos publicados entre 1955 y 1967 y recogidos en el volumen de recopilación Studies in Politics, Philosophy and Economics: «Degrees of Explanation», «The Theory of Complex Phenomena» $\mathrm{y}$ «Notes on the Evolution of Systems of Rules of Conduct». Estos tres trabajos pueden entenderse como desarrollos parciales de un mismo argumento y pueden leerse, de hecho, como una unidad. En este argumento, con independencia de las consideraciones metodológicas a que pueda dar lugar (y que no son objeto de este trabajo) se pueden encontrar de forma explícita argumentos, referencias a propiedades e hipótesis que guardan una estrecha relación con los desarrollados en

11 La diversidad viene dada por la propia dinámica de las personas que componen esa sociedad, que es única e irrepetible: «En los órdenes espontáneos nadie conoce - ni precisa conocer - cuantos detalles afectan a los medios disponibles o a los fines perseguidos. Tales órdenes se forman a sí mismos. Las normas que facilitan su funcionamiento no fueron apareciendo porque los distintos sujetos llegaran a advertir la función de las mismas, sino porque prosperaron en mayor medida aquellos colectivos que, sometiéndose a ellas, lograron disponer de más eficaces esquemas de comportamiento» (Hayek 1997: 209) 
la literatura de la no-linealidad. Por esta razón debemos detenernos minuciosamente en lo que en ellos se dice.

«Degrees of explanation» es el que primero aparece cronológicamente (1955) y también donde encontramos el punto de partida del argumento que une a los tres. Éste se puede resumir de la siguiente forma: Hayek reconoce que la discusión del método científico ha estado tradicionalmente asociada al ejemplo de la física clásica, debido a su elevado grado de desarrollo, dando lugar a un proceso de imitación de su método por el resto de la ciencias (1967: 3). Hayek no cuestiona en ningún momento estos métodos para la física. El problema metodológico se presenta, a su juicio, cuando intentamos aplicar este método a ciencias y disciplinas cuyo campo de análisis no se ajusta al de la física teórica. Éste se define, según Hayek, en el campo de la física, como «la totalidad de los fenómenos donde el número de variables de diferentes tipos significativamente conectadas es suficientemente pequeño [y] nos permite estudiarlos como si formasen un sistema cerrado para el que podemos observar y controlar todos los factores determinantes; y podríamos haber sido conducidos a tratar ciertos fenómenos como fuera del objeto de estudio de la física precisamente porque no es este el caso» (Hayek 1967: 3-4).

El problema, según Hayek, es que no se ha apreciado suficientemente el carácter de los fenómenos del segundo tipo que menciona. Y a esta apreciación dedica el resto de este trabajo. Para Hayek, las disciplinas científicas que caen dentro de este segundo tipo no sólo son las ciencias sociales. Las ciencias aplicadas, como la oceanografía y la geología, también caen dentro de este tipo (Hayek 1967: 6). ¿Qué es lo que no se ha apreciado suficientemente en el tipo de fenómenos que estudian estas disciplinas? Sencillamente, que «el número de variables significativamente interdependientes es muy grande y sólo una parte de ellas puede ser observada en el práctica» (Hayek 1967: 8). Es decir, nos encontramos, de forma característica, con situaciones 
complejas. Ante estas situaciones, nuestros conocimientos sobre la operación de determinadas fuerzas en entornos sencillos (como puede ser el caso de las leyes físicas en el caso de la geología) no nos permite conocer con certeza si se siguen manteniendo en situaciones complejas (p. 10), dado que no tenemos medios de contrastar este supuesto, porque la dificultad estriba, precisamente en que «somos incapaces de averiguar mediante la observación la presencia y la ordenación específica de la multiplicidad de factores que forman el punto de partida de nuestro razonamiento deductivo» (Hayek 1967: 10). Lo único que podemos obtener con nuestra explicación es una clasificación de los tipos de eventos que se pueden esperar y de los que no, debido a la operación de las fuerzas consideradas. Conocimiento que no debe considerarse como trivial, por cuanto nos ofrece «nichos» en los cuales es posible clasificar nuevas observaciones e indicar las direcciones posibles en las que podemos esperar que los fenómenos varíen (p. 11). Como ejemplo de esta «explicación del principio» (sic), Hayek cita la teoría de la evolución mediante la selección natural de los diferentes organismos. Entre otras muchas cosas, esta teoría clasifica los hechos entre "permitidos» y «prohibidos», es decir, entre aquellos que son esperables y los que no. Las predicciones de esta teoría se refieren a las propiedades resultantes de un tipo de fenómenos y no de fenómenos particulares, del mismo modo que es posible formular modelos matemáticos que, con toda seguridad, serán imposibles de resolver para valores concretos, lo que no obsta para que esos modelos tengan un significado teórico claro y preciso. ${ }^{12}$

12 «Si consideramos un modelo formal consistente en un sistema de ecuaciones algebraicas o «ecuaciones preposicionales», contendrá afirmaciones acerca de la estructura de relaciones incluso si no sabemos el valor de ninguna de las variables, e incluso si tenemos sólo la información más general acerca del carácter de las funciones que ocurren en él: excluirá la posibilidad de aparición de ciertas combinaciones de valores en cualquier fenómeno que el modelo afirme representar; nos dirá tanto las combinaciones de variables que pueden ocurrir en cualquier momento del tipo como la gama de valores que otras variables pueden 
El siguiente paso del argumento se publica unos cuantos años después, en 1964. "The Theory of Complex Phenomena» es, como indica el título, una teoría acerca de la forma de acceder a fenómenos que, en «Degrees of Explanation» se habían catalogado como «fuera» del ámbito de aplicación de la metodología de la física teórica. "The Theory of Complex Phenomena» comienza casi donde terminó «Degrees of Explanation». El primer apartado se titula «Pattern Recognition and Pattern of Prediction", donde diferencia entre la predicción de hechos concretos de acuerdo con una pauta de la realidad definida por la teoría, y el reconocimiento de una pauta que sólo puede predecir clases de fenómenos. Es decir, entre el uso de esas pautas teóricas en el campo de los fenómenos simples y los fenómenos complejos. ${ }^{13}$ Y lo que a su juicio parece evidente es que, a medida que nos desplazamos desde los fenómenos inanimados a los fenómenos animados y sociales, la complejidad aumenta. Pero incluso en los fenómenos aparentemente más simples, según su criterio, se producen órdenes que superan el ámbito de la mecánica clásica: «Por otro lado, incluso elementos de los fenómenos biológicos (o cibernéticos) tan relativamente simples como los sistemas de retroalimentación, en los que ciertas combinaciones de estructuras físicas producen una estructura global que posee propiedades características distintas, requieren para su descripción algo mucho más elaborado que algo que las leyes generales que describen la mecánica» (Hayek 1967: 26).

asumir cuando una o más variables sean conocidas. Por supuesto, en la medida en que seamos capaces de insertar más y más valores concretos para las variables, esta gama se irá estrechando hasta que alcancemos el punto en que el sistema está completamente determinado y sólo existe un valor posible para la variable restante» (Hayek 1967: 15).

${ }^{13}$ Hayek define en la p. 25 los fenómenos complejos de forma más precisa que en «Degrees of Explanation». El criterio para decidir el grado de complejidad de un tipo de pautas se define por el número mínimo de elementos en los que debe consistir una pauta para mostrar todos los atributos característicos de los que consiste el tipo o clase de pautas a las que pertenece. 
Obsérvese la extraordinaria similitud entre esta afirmación y el moderno concepto de autoorganización: propiedades de la estructura distintas de las de los componentes. Pero no sólo con la autoorganización. Un poco más adelante, Hayek escribe: «La «emergencia» de «nuevas» pautas como resultado de un aumento en el número de elementos entre los que existe una relación simple, implica que esta mayor estructura en su conjunto poseerá ciertas características generales o abstractas que aparecerán recurrentemente con independencia de los valores particulares de los datos individuales, en tanto en cuanto se preserve la estructura general (descrita, por ejemplo, por una ecuación algebraica.) Tales «conjuntos», definidos en términos de ciertas propiedades generales de su estructura, constituirán objetos distintos de explicación para una teoría, incluso aunque esa teoría no sea más que un modo de ajustar enunciados sobre las relaciones entre los elementos individuales» (Hayek 1967: 26).

Aquí tenemos la clave de la teoría de los órdenes espontáneos de Hayek. El aumento en el número de elementos componentes del sistema implica la aparición de nuevas propiedades de la estructura, que se constituyen así en objetos específicos de estudio al margen de sus partes componentes. Porque lo que le interesa a Hayek son precisamente las pautas complejas que van dando lugar a propiedades «que producen el automantenimiento de la estructura que las muestra».

El capítulo 3 de los Studies se titula «Rules, Perception and Intelligibility», y aquí Hayek trata con cierta profundidad los procesos de adquisición del conocimiento por parte las personas. A partir de los análisis de psicología teórica ya desarrollados en su Sensory Order, Hayek explica cómo las personas adquieren pautas o hábitos de conducta a partir de la interacción con la realidad y con otros agentes. La explicación es meramente fisiológica, por cuanto se trata de propiedades que se derivan de la evolución del orden mental a partir de la interacción de la persona con la realidad. Se trata, a los efectos del argumento que 
nos ocupa, de un paso intermedio, por cuanto Hayek quiere explicar el comportamiento de las «partes»o «elementos», esto es, las personas, que componen la estructura $u$ orden social que se define como orden complejo. ${ }^{14}$ Una vez realizado, llegamos al capítulo 4, que constituye la parte final del argumento.

El objetivo de «Notes on the Evolution of Systems of Rules of Conduct. The Interplay between Rules of Individual Conduct and the Social Order of Actions» queda perfectamente claro desde el primer párrafo. El objetivo de estas notas - dice Hayek- es «dejar clara la importante distinción entre los sistemas de reglas de conducta que gobiernan el comportamiento de los miembros individuales de un grupo (o los elementos de cualquier orden) por un lado, y por el otro, el orden o la pauta de las acciones que resultan de este grupo en su conjunto» (Hayek 1967: 66).

En la nota 1 Hayek define lo que entiende por «orden social» como una estructura de acciones de todos los miembros del grupo. Hayek supone que, de una forma y otra (cultural o genéticamente) las pautas de comportamiento individual se transmiten dentro del grupo (animal o humano). Queremos llamar la atención sobre un hecho que muchos críticos de Hayek parecen pasar por alto. No todos los órdenes sociales son posibles, porque no todo sistema de reglas de comportamiento puede producir un orden. Esto no es sorprendente. Lo que sorprende es la argumentación que Hayek utiliza: «No todo sistema de reglas de conducta individual producirá un orden global de las accio-

${ }^{14}$ Hayek también se refiere a la mente como fenómeno complejo. El hecho de que no nos detengamos en esta explicación se debe, sencillamente, a que no aporta nada a la relación de las modernas teorías de la complejidad tal y como la estamos abordando aquí. No obstante, remitimos al lector interesado, además de este trabajo y al Sensory Order, a Weimer, W. y Palermo, D. (1982) vol. 2. En particular, los capítulos 12 («Hayek's Approach to the Problems of Complex Phenomena: An Introduction to the Theoretical Pscychology of The Sensory Order»), el capítulo 13, del propio Hayek, («The Sensory Order After 25 years») y capítulo 15 («WeimerHayek Discussion). Gray (1994) también contiene importantes apreciaciones, en especial capítulos 1 y 2. 
nes de un grupo de individuos; y que un sistema dado de normas de conducta individual producirá un orden de acciones, y el tipo de orden, dependerá de las circunstancias en las que un individuo actúa. El ejemplo clásico en el que la propia regularidad de los elementos produce "perfecto desorden» es la segunda ley de la termodinámica, el principio de entropía. Es evidente que en un grupo de muchos seres vivos el orden de acciones se producirá no sólo por las múltiples reglas de conducta individual, sino también por las circunstancias en que viven» (Hayek 1967: $67)$.

Aunque, por supuesto, no hay ninguna referencia al trabajo de Prigogine, el argumento discurre por líneas parecidas: la transmisión cultural de las pautas entre las personas en gran medida se produce individuo a individuo. Obviamente, el problema se plantea siguiendo el argumento de «The Theory of Complex Phenomena»: identificar qué tipo de sistemas producen orden social y cuáles desorden. Previamente, Hayek realiza una serie de consideraciones para diferenciar entre el orden de las acciones de un grupo y las reglas de comportamiento de los individuos (Hayek 1967: 68-69). Entre ellas, cabe destacar las siguientes: a) Se puede analizar el orden de las acciones sin tener en cuenta el comportamiento de los individuos que lo componen, siendo teóricamente posible que el mismo orden sea producido por diferentes pautas de comportamiento individuales. b) Bajo ciertas circunstancias, es posible que el mismo conjunto de normas individuales produzca un cierto tipo de orden de acciones, pero no lo hará si esas circunstancias cambian. c) Un cierto tipo de orden puede contribuir de la misma forma a la supervivencia de los miembros del grupo con independencia de las normas particulares de conducta individual que se presenten. Finalmente, d) la diferencia entre la ordenación del conjunto y la regularidad de las acciones individuales se manifiesta en que el conjunto puede ordenarse sin que la acción de un elemento concreto muestre cierta regularidad (como es el caso de la plani- 
ficación centralizada). Todo esto, junto con otras propiedades que no vienen al caso que estamos estudiando, le lleva a concluir que:

«El orden conjunto de acciones en un grupo es en dos aspectos más que la totalidad de las regularidades observables en la acciones de los individuos y no pueden ser completamente reducidos a ellos. Esto es así no sólo en el sentido trivial en el que un todo es más que la mera suma de sus partes, sino que presupone también que estos elementos están relacionados unos con otros de una determinada manera. Es también más porque la existencia de tales relaciones que son esenciales para la existencia del conjunto no pueden ser explicadas en su totalidad por las interacciones de las partes, sino sólo en sus interacciones con un mundo exterior tanto para las partes individuales como para el todo. Si existen estructuras persistentes y recurrentes de un cierto tipo (esto es, mostrando cierto orden), esto es debido a los elementos que responden a las influencias externas que es probable que se encuentren, de tal forma que esto lleve a la preservación o restauración del orden; y de esto, además, pueden depender las oportunidades de los individuos de preservarse a sí mismos».

(Hayek 1967: 70-71)

Esta conclusión es algo que se desprende directamente de las consideraciones hechas en los trabajos previos. La pauta del comportamiento del sistema consiste en el sistema de relaciones de los elementos, cuyas circunstancias concretas de comportamiento son imposibles de averiguar, dada la complejidad de las mismas en su ubicación espacio-temporal. La estructura se mantiene porque los elementos se comportan de algún modo que hace que ésta se mantenga aun cuando cambien dichas circunstancias particulares. Esto le lleva directamente a la consideración de la relación del orden con el resto del mundo, sin que, en ningún momento, se trate de un sistema cerrado. Todo lo contrario; como consecuencia de la complejidad del fenómeno a estudiar, lo único que se puede afirmar es que la pauta de comportamiento del orden se mantiene (lo que es observable) sin que sea posible apreciar la causalidad del fenómeno con el grado de preci- 
sión de los fenómenos «simples» de la física. Las consecuencias siguen manteniendo un asombroso parecido con las estructuras disipativas de Prigogine: «A partir de cualquier conjunto de reglas de conducta de los elementos sólo surgirá una estructura estable (mostrando un control «homeostático») en un entorno en el que prevalezca una cierta probabilidad de encontrar la clase de circunstancias a las que las reglas de conducta están adaptadas. Un cambio en el entorno puede exigir, para que perdure el conjunto, un cambio en el orden del grupo y por tanto de las reglas de conducta individual, de tal modo que el orden resultante pueda permitir al grupo persistir en circunstancias que, sin tales cambios, le hubiera enviado a su destrucción» (Hayek 1967: 71).

Este control homeostático no implica que las nuevas normas que surjan lleven necesariamente a la conservación del grupo. Existe un proceso evolutivo de selección de aquellas normas que aumentan la eficiencia del grupo y las que lo disminuyen. En este sentido cabe entender el criterio demográfico como medida de la eficacia del grupo, argumento que aparecerá con profusión en La fatal arrogancia. La nueva norma de comportamiento será efectiva dentro del conjunto concreto de circunstancias en que operan los individuos del grupo y del resto de las normas que se siguen manteniendo. En ocasiones, y bajo unas condiciones concretas, esta norma puede aumentar la eficacia. Bajo otras circunstancias, puede ocurrir todo lo contrario, con lo que la eficacia global del grupo disminuirá. Obsérvese que Hayek sigue, punto por punto, el argumento desarrollado en los trabajos anteriores. La conclusión metodológica que se desprende de esto para las ciencias sociales, es, a juicio de Hayek, inequívoca: «Toda la tarea de la teoría social consiste en poco más que un esfuerzo por reconstruir los órdenes conjuntos así formados, y las razones por las cuales es necesario ese aparato especial de construcción conceptual que representa la teoría social es la complejidad de esta tarea. Debe quedar claro también que tal 
teoría de las estructuras sociales puede ofrecer sólo una explicación de ciertos y altamente abstractos rasgos de los diferentes tipos de estructuras (o sólo en los «aspectos cualitativos»), debido a que estos rasgos abstractos serán todos aquellos predecibles o que proporcionan una guía útil para la acción» (Hayek 1967: 71-72).

Es decir, que la teoría social sólo puede producir predicciones generales acerca de dos tipos de fenómenos, cuyo criterio de diferenciación es si se produce aumento de la eficiencia del grupo o no. A juicio de Hayek, sólo la teoría económica y la lingüística han producido teorías de este tipo. Hayek señala aquí también uno de los argumentos que desarrollará en trabajos posteriores, fundamentalmente en Derecho, legislación y libertad (Hayek, 1973/79) y que se resumirán en La fatal arrogancia, a saber: si pueden alterarse las normas de comportamiento individual de forma que aumente la eficiencia del grupo. La opinión de Hayek es de sobra conocida y remitimos al lector interesado en profundizar en ella a esas obras. ${ }^{15}$

Podemos concluir este apartado diciendo que, sin ningún género de dudas, existen claras similitudes formales y conceptuales entre la teoría de los órdenes espontáneos de Hayek y los conceptos desarrollados en la literatura de los sistemas dinámicos no lineales. Sin que se pueda afirmar con rotundidad que Hayek fuese consciente de los desarrollos incipientes de esta literatura, sí es posible observar una serie de rasgos y propiedades comunes más allá de lo que, en principio, cabría esperar.

15 Una excelente exposición de este asunto es Socialismo, cálculo económico y función empresarial, de Jesús Huerta de Soto (Huerta de Soto, 1992). 


\subsection{La dependencia de la trayectoria}

A diferencia del orden espontáneo, la propiedad de la dependencia de la trayectoria, como la idea de la irreversibilidad, resulta más difícil de encontrar en los textos de los autores austriacos. Y tampoco se puede decir que los autores austriacos contemplasen como necesario el uso de esta propiedad; más bien, se puede decir que aparece en sus teorías allí donde la explicación de determinadas leyes o fenómenos económicos la cumple. Entre las diversas teorías especiales de la Escuela Austriaca, se pueden encontrar al menos dos en las cuales aparece esta propiedad de una forma más evidente: son las teorías del surgimiento espontáneo de las instituciones - en particular del dinero-, la teoría del dinero y la teoría del capital. Esto no obsta para que, en otros contextos, se pueda también encontrar. Pero su documentación es bastante más imprecisa.

Si bien, como argumentábamos en el apartado anterior, resulta difícil extraer una idea clara de la dependencia de la trayectoria dentro de la teoría del surgimiento espontáneo de las instituciones, parece evidente que la teoría del origen del dinero - como teoría especial dentro de aquélla- hace depender la evolución de éste de las condiciones iniciales - históricas- de su surgimiento. El dinero como medio de pago surge allí donde las condiciones de mercado lo permiten. En particular, surge cuando, dentro de un mercado de trueque, un bien adquiere el carácter de medio de pago. Este proceso tiene como condición que el bien en partículas tenga la propiedad de la liquidez, esto es, que tenga un valor estable y que se pueda adquirir y enajenar con facilidad en un mercado de trueque. Esto es, que sea un bien de amplia aceptación por los agentes implicados. Así se originaron multitud de medios de pago en la antigüedad - cabezas de ganado, sal, cacao, etc.- - De este hecho no se puede extraer que Menger (1873), autor de esta teoría, contemplara la dependencia de la trayectoria como propiedad de este proceso. Un 
bien puede considerarse, en esta situación, medio de pago mientras se mantengan determinadas circunstancias de mercado - en particular que sea un bien fácilmente intercambiable en los mercados-. En el momento en que esas circunstancias cambian, cambiaría también la forma material del medio de pago: otros bienes serían candidatos a convertirse en medios de pago, si es que este proceso puede operar bajo las nuevas circunstancias. Pero, en el momento en que se intenta racionalizar el proceso ulterior hasta el surgimiento del papel-moneda, sí se puede observar la dependencia del papel respecto del bien físico que hasta ese momento se utilizaba como medio de pago. El origen de los billetes de banco se puede rastrear hasta el momento histórico en el cual los orfebres y la banca incipiente comienzan a emitir resguardos sobre los depósitos de oro. Lo relevante aquí no es que del oro se pasase al papel. Lo relevante es que sin el uso previo de un bien material no se puede pasar al uso del papel. Y lo mismo se puede decir respecto del papel a las actuales transacciones electrónicas de medios de pago. Es decir, la evolución de los medios de pago es dependiente del curso de los acontecimientos y no puede decirse que sea posible crear un nuevo medio de pago sin referencia alguna a los previamente existentes. Sin embargo, no se puede decir que esta propiedad tenga la generalidad suficiente como para decir que se deduce de forma exacta de una clara dependencia de la trayectoria. En particular, se dice que depende de la existencia de un bien físico en particular utilizado como medio de pago, pero no se dice que un determinado medio de pago fiduciario dependa inexorablemente de un medio de pago físico concreto. Más que de dependencia de la trayectoria se puede hablar de vínculo causal en la evolución y nada más. Pero esta relación causal tiene un problema específico que sí tiene de forma explícita una dependencia de la trayectoria. Se trata de problema del valor en la teoría monetaria austriaca.

Al estudiar los problemas de la teoría del valor del dinero, Mises se encontró con el siguiente argumento circular. La teoría 
del precio de los bienes, en su formulación más general, nos dice que el precio de un bien depende de la oferta y la demanda del mismo. La utilidad marginal que reporta ese bien para los diferentes agentes junto con las posibilidades técnicas de producción del mismo son las causas que explican el precio de ese bien. Si aplicamos este esquema a la teoría monetaria, continúa Mises, y dejamos de lado por un momento los problemas de la oferta de dinero, resulta que el valor del dinero dependerá de la demanda del mismo. Pero resulta que la demanda de dinero depende del valor de ese dinero en los mercados, esto es, de su valor de cambio, que es lo que queremos determinar. Para solucionar la circularidad, Mises enunció el teorema de la regresión, según el cual el valor del dinero depende de su demanda, que a su vez no depende del valor de cambio en ese momento, sino del valor de cambio de la moneda en un periodo anterior. Resulta así que el valor del dinero a día de hoy depende del valor que tuvo en el momento en que ese preciso medio de pago vio la luz. Como la transición de un papel moneda a otro ha de hacerse, necesariamente, a través de su conversión respecto de la moneda previamente existente, el proceso nos llevaría hasta el momento en que el primer papel-moneda vio la luz. Con independencia de las consideraciones y críticas que se puedan hacer a esta teoría monetaria, resulta evidente que exhibe de forma explícita la dependencia de la trayectoria. Pero, al contrario que en la teoría del surgimiento del dinero, donde esta propiedad se manifiesta de forma contingente, aquí lo que se dice es que el valor del dinero depende históricamente del valor del bien físico sobre el que se produjo la conversión a medio de pago. El valor del dinero no depende de forma contingente de valor del medio de pago; resulta que no se puede explicar el valor sin trazar toda la secuencia hasta el momento inicial.

Todavía más claramente que en la teoría monetaria, se puede apreciar la presencia de esta propiedad en la teoría austriaca del capital. Aquí nos vamos a encontrar con que para los autores 
austriacos, el capital consiste en un entramado de planes ${ }^{16}$ orientados a la producción de bienes de consumo en un futuro más o menos cercano. Estos planes «consumen» tiempo, esto es, producir exige un periodo de tiempo que se puede acortar o alargar en función de las disponibilidades de bienes de capital (que se pueden adquirir en los mercados o producirse en el mismo proceso). Pero, además de consumir tiempo, estos planes consisten en una ordenación de las acciones a tomar. Esta ordenación puede tener un carácter definitivo. Pero también puede ser posible la alteración a medida que el proceso de producción se va completando tal y como estaba previsto en el plan. El que esta alteración se pueda producir, dependerá de la presencia de bienes de capital no complementarios o no específicos para ese proceso productivo. Si la mayoría de los bienes son de este tipo, entonces puede resultar sencillo modificar el plan e, incluso, terminar produciendo bienes de consumo distintos de los inicialmente previstos (o que dichos bienes de capital se vendan a productores de bienes de consumo distintos de los que a priori estaban más necesitados de estos bienes). Pero si, por el contrario, los bienes de capital utilizados son altamente específicos, entonces resultará difícil modificar la ordenación inicial y, por consiguiente, el resultado final del proceso será altamente dependiente de las decisiones que se tomaron en los primeros momentos. Determinadas decisiones implicarán que en un estadio más avanzado del proceso las opciones abiertas para su modificación puedan ser radicalmente distintas de las que quedarían si se hubieran tomado otras decisiones distintas. En este sentido, el que el proceso productivo muestre, en un momento $t$, cierta configuración, dependerá de las decisiones que se tomaron inicialmente y, en este caso, será sensible a las condiciones iniciales.

La mera existencia de bienes de capital complementarios hace que los procesos productivos exhiban dependencia de la trayec-

\footnotetext{
${ }_{16}$ Véase Kirzner 1966. En especial el capítulo 1.
} 
toria. Producir lleva tiempo y a medida que el proceso se va completando, la posibilidad de cambiar de proceso como consecuencia de una alteración en, por ejemplo, el precio del bien que se pretende producir, se reduce a medida que se llega a las etapas finales. Las decisiones o los cambios en la utilización de las técnicas productivas en los últimos estadios de producción son altamente dependientes de las etapas que ya se han completado. En ese momento, es posible que se revele como más eficiente otra técnica diferente como consecuencia de un cálculo erróneo de las cantidades de factores no específicos a utilizar, o de un cambio en su valor. Sin embargo, en la medida en que la elevada complementariedad del proceso no permite en ese momento alterar a voluntad la utilización de los factores productivos, la técnica finalmente utilizada será la más eficiente dadas las circunstancias, esto es, dados los pasos previamente completados en los procesos de producción previos. Esto no obsta para que, una vez que el proceso está finalmente completado, las pérdidas hagan que la empresa tenga que cerrar y se vea sustituida por otras que, utilizando diferentes técnicas, sean capaces de generar beneficios. Pero puede perfectamente ocurrir también que una posición dominante en ese mercado haga que la empresa pueda seguir produciendo con la técnica original. Pero esto sólo se puede producir en el caso en que la elevada complementariedad de los factores intermedios de producción haga imposible el cambio en la técnica de producción. Y, por supuesto, bajo la hipótesis de que la competencia de otras empresas no tenga intensidad suficiente como para desplazarla del mercado.

\section{Conclusiones}

Los resultados de este análisis son bastante concluyentes. En primer lugar, constatamos que existe una similitud formal —que no sustantiva- entre propiedades de los procesos sociales deducidas por los clásicos de la Economía Austriaca y las de los 
sistemas dinámicos no-lineales. En segundo lugar, que tal similitud no permite hablar ni de una equiparación de tales propiedades en sentido sustantivo ni de una aplicación directa de la modelización no-lineal a procesos austriacos; pero que, en cualquier caso, se trata de una similitud que plantea cuestiones de gran interés para la metodología científica y para la teoría económica en general, puesto que es obvio que ni Menger ni Mises ni Hayek buscaban una analogía deliberada con los sistemas nolineales, pese a que podamos suponer que el último si estaba, al menos, informado de la teoría de sistemas de von Bertalanffy.

Téngase en cuenta que, por un lado, si nos instalamos en una posición metodológicamente monista y continuamos utilizando como ciencia de referencia la física, en ese caso muchas de las hipótesis y formas de modelizar que viene utilizando habitualmente la economía deben empezar a ser revisadas. Esta dirección es la que están siguiendo nuevos desarrollos, como la economía de los agentes computacionales o la introducción de modelos no-lineales para formalizar la explicación de procesos tales como la difusión de la innovación o el crecimiento económico. No obstante, y con ser desarrollos de gran interés, existe un problema de fondo en estas líneas de avance: que no pueden dar cuenta de todos aquellos procesos económicos en los que interviene la intencionalidad humana. Pueden replicar los resultados de tales procesos, pero siempre existirá una parte del proceso que no podrán explicar. Creemos, sin embargo, que pueden ser de gran utilidad para explicar procesos económicos analíticos en entornos locales.

Pero si nos instalamos en una metodología dualista, los resultados del análisis que hemos llevado a cabo aquí abren un nuevo capítulo en la ya larga polémica sobre el uso de las matemáticas en las ciencias sociales en general y económicas en particular. Porque se ha criticado mucho desde esta perspectiva el uso de herramientas matemáticas «importadas» de otros campos de la ciencia. Pero no se ha preparado el terreno para la operación 
contraria, esto es, el diseño de unas matemáticas que se ajusten a las necesidades de la ciencia social. Y el caso que aquí nos ocupa demuestra de forma efectiva esta posibilidad; no de forma directa, evidentemente, pero sí de forma indirecta. La similitud formal entre las propiedades deducidas dejan abierta la posibilidad - por remota que pueda parecer- de que empecemos a pensar en diseñar instrumentos matemáticos que se ajusten a las necesidades teóricas de las ciencias sociales y, en particular, a las de la teoría económica, en lugar de proceder a importaciones de sistemas de razonamiento formal que, por la propia naturaleza del análisis para el que fueron diseñados, difícilmente pueden dar cabida a las hipótesis de comportamiento de nuestro objeto de estudio.

\section{REFERENCIAS BIBLIOGRÁFICAS}

ARROW (1998): «Economic Theory and the Hypothesis of Rationality», en Eatwell, J., Milgate, J.M., Newman, P. (eds), The New Palgrave: A Dictionary of Economics, vol. 2, Londres y Nueva York: Macmillan.

AUYANG (1998): Foundations of Complex-System Theories in Economics,

Evolutionary Biology, and Statistical Physics, Cambridge: Cambridge University Press.

GroeneWEGEN, VROMEN (1997): «Theory of the firm revisited», en

Magnusson, L., Ottoson, J. (eds), Evolutionary Economics and Path-Dependency, Cheltenham: Edward Elgar.

HAYEK (1952): The Sensory Order. An Inquiry into the Foundations of

Theoretical Psychology. Londres y Henley: Routledge \& Kegan

Paul. [Trad. esp.: El orden sensorial, Madrid: Unión Editorial, 2003].

HAYEK (1967): Studies in Philosophy, Politics and Economics, Chicago:

University of Chicago Press.

HayeK (1978): New Studies in Philosophy, Politics, Economics and the History of Ideas. Londres: Routledge \& Kegan Paul. 
HAYEK (1988): The Fatal Conceit. The Errors of Socialism. Chicago: The University of Chicago Press.

Hodgson, G. (1993): Economics and Evolution, Ann Arbor: University of Michigan Press.

HuERTA DE SOTO (1992): Socialismo, cálculo económico y función empresarial. Madrid: Unión Editorial.

KiRZNER: (1966): An Essay on Capital, Nueva York: Augustus M. Kelley.

LORENZ: (1993): Nonlinear Dynamical Economic and Chaotic Motion. Berlín-Heidelberg-Nueva York: Springer-Verlag

MENGER: (1981): Principles of Economics, Nueva York y Londres: New York University Press

Mises: (1996): The Human Action. A Treatise on Economics. $4 .{ }^{\text {th }}$ Edition, San Francisco: Fox \& Wilkes. [Trad. esp.: La acción humana, 6. ${ }^{a}$ ed., Madrid: Unión Editorial, 2001].

MiROWSKI (1989): More Heat than Light: Economics as Social Physics, Physics as Nature's Economics. Cambridge y Nueva York: Cambridge University Press.

Nieto, U. (1988): Historia del tiempo en economía, Madrid: McGrawHill

PRIGOGINE (1993): «Bounded Rationality: From Dynamical Systems to Socio-economic Models», en Day, R., Chen, P. (eds.), Nonlinear Dynamics \& Evolutionary Economics, Nueva York \& Oxford: Oxford University Press.

RIZELLO (1997): «The Microfoundations of Path- Dependency», en Magnusson, L., Ottoson, J. (eds), Evolutionary Economics and Path-Dependency, Cheltenham: Edward Elgar.

Weimer \& PALERMO (1982): Cognition and the Symbolic Processes, vol.

2, Hillsdale: Lawrence Erlbaum Associates.

WIT (1985): «Coordination of Individual Economic Activities as an Evolving Process of Self-Organization», Economie Appliquée XXXVII n. ${ }^{\circ} 3 / 4$. 